\title{
A pearl-chain-like anode composed of silicon- porphyrin hits peaks in lithium-ion capacity
}

\author{
Wen-Yin Ko, ${ }^{1,}{ }^{*}$ Man-Jyun Fang, ${ }^{1}$ Meng-Shan Li, ${ }^{1}$ Wei-Cheng Hsu, ${ }^{1}$ Yu-Wei Huang, ${ }^{2}$ Chang-Wei \\ Cheng, ${ }^{3}$ Bing-Joe Hwang, ${ }^{2}$ Shangjr Gwo, ${ }^{3,4}$ and Kuan-Jiuh Lin ${ }^{1, *}$ \\ 1.Department of Chemistry, National Chung Hsing University, Taichung 402, Taiwan \\ 2.NanoElectrochemistry Laboratory, Department of Chemical Engineering, National Taiwan \\ University of Science and Technology, Taipei 106, Taiwan \\ 3.Department of Physics, National Tsing-Hua University, 300 Hsinchu, Taiwan \\ 4. Research Center for Applied Sciences, Academia Sinica, Nankang, Taipei 115, Taiwan
}

\section{E-mail: wyko@nchu.edu.tw; kjlin@nchu.edu.tw}

\section{Experimental Section}

\section{Methods}

Materials synthesis. The silicon-porphyrin pearl-chain-like framework was prepared directly onto the surface of the $\mathrm{TiO}_{2}$ bundled nanowire modified $\mathrm{Cu}$ foil through a simple step-by-step vapor deposition process. The $\mathrm{TiO}_{2}$ bundled nanowire modified $\mathrm{Cu}$ foil was performed through the process similar with analogous procedure as described in our previous work: ${ }^{1}$ firstly, titanium film with a thickness of approximately $50 \mathrm{~nm}$ was sputter-deposited onto the surface of Cu foil which is preadhesive to glasses surface $\left(1.5^{*} 1.5 \mathrm{~cm}\right)$ to form a thin Ti-sputtered Cu foil through a magnetic sputter instrument (TVC-M8C8TV, Transvac co. Ltd.) equipped with a Ti target at a sputter rate of 4 $\mathrm{nm} / \mathrm{s}$ with an Ar flow rate of $300 \mathrm{sccm}$. The distance between sputtering target and substrate was $90 \mathrm{~cm}$. The background pressure was $8.5 \times 10^{-7}$ Torr and the sputtering pressure was $1.2 \times 10^{-3}$ Torr. The Ti-sputtered $\mathrm{Cu}$ foil was subsequently heated at $80{ }^{\circ} \mathrm{C}$ for $2 \mathrm{~h}$ under an alkali condition containing a $5 \mathrm{M} \mathrm{NaOH}$ (ACS reagent, $\geq 97.0 \%$, Sigma-Aldrich) aqueous solution. Afterwards, a 3D porous $\mathrm{TiO}_{2}$ nano-scaffolds featuring bundled $\mathrm{TiO}_{2}$ cross-linked nanowires (TBNW) was formed directly onto the surface of $\mathrm{Cu}$ foil and subsequently washed by ethanol several times and then dried at $50{ }^{\circ} \mathrm{C}$ overnight. Then, the Si and tetrakis(4-carboxyphenyl) porphyrin (TCPP) materials were vacuumdeposited onto the surface of the TBNW framework to form a unique silicon-porphyrin pearl-chainlike framework. In this process, the Si metal was firstly sputter-deposited onto the surface of the TBNW framework with a deposition time of 3 min through an RF-magnetron sputtering system (TVCMR6C4TV, Transvac co. Ltd.) at a sputter rate of $1.8 \mathrm{~nm} / \mathrm{s}$ with an Ar flow rate of $150 \mathrm{sccm}$ where the background pressure was $8 \times 10^{-6}$ Torr and the sputtering pressure was $2.37 \times 10^{-3}$ Torr and 
subsequently, TCPP layer with $10 \mathrm{~nm}$ in thickness was deposited by thermal evaporation system under $10^{-6}$ Torr pressure with $0.05 \AA$ /s deposition rate. Afterwards, the prepared films were rinsed and immersed in acetone for $24 \mathrm{hr}$ to remove the Cu foil electrodes from the glass substrate. Finally, a 3D hierarchically interconnected porous nano-scaffolds based self-standing anode composed by silicon-porphyrin pearl-chain-like framework coated onto $\mathrm{TiO}_{2}$ bundled nanowire decorated $\mathrm{Cu}$ foil was obtained (denoted as TCPP/Si/TBNW). The typical mass loading of the active material of TCPP/Si composite was $\sim 0.05 \mathrm{mg} / \mathrm{cm}^{2}$.

Material characterizations. The morphology and the thickness of the samples were analyzed, using a Zeiss Ultra-Plus field emission scanning electron microscope (FESEM) with an accelerating voltage of $3 \mathrm{kV}$. Measurements of Raman spectra were carried out by a Raman microspectroscopy system of Nanofinder 30 (Tokyo Instruments, Inc.) with an excitation source of $\mathrm{He}-\mathrm{Ne}$ laser ( $\lambda$ ex=632.8 $\mathrm{nm}$ ). A high-resolution transmission electron microscope (HRTEM) operated was also applied for the microstructural investigation of the obtained products using a JEM-2100F at $200 \mathrm{kV}$.

Electrochemical characterizations. Electrochemical measurements of the prepared anode materials were tested by assembling the CR-2032 coin-type cell in an argon-filled glovebox system (MBraun, Germany) with $\mathrm{O}_{2}$ and $\mathrm{H}_{2} \mathrm{O}$ level maintained below $1 \mathrm{ppm}$ with Li metal as a counter electrode, a Celgard 2325 membrane with $19 \mathrm{~mm}$ as the separator, and $1 \mathrm{M} \mathrm{LiPF}_{6}$ in a mixture of ethylene carbonate $(E C) /$ diethyl carbonate (DEC) with the volume ratio of 1 as the electrolyte. The electrochemical performances were conducted on a battery test system (Automatic battery cycles WBCS3000 M1) at room temperature in the voltage window of $0-3.0 \mathrm{~V}$ versus $\mathrm{Li} / \mathrm{Li}^{+}$at different current densities after $3 \mathrm{~h}$ rest. The resulting data of charge/discharge capacity for TCPP/Si/TBNW anodes were obtained by deducting the TBNW capacitance from the total. Cyclic voltammetry and the electrochemical impedance spectroscopy (EIS) measurements were performed through an electrochemical workstation (CHI 611E) between $0 \mathrm{~V}$ and $3.0 \mathrm{~V}$ versus $\mathrm{Li} / \mathrm{Li}^{+}$at a scan rate of $0.1 \mathrm{mV} / \mathrm{s}$. 


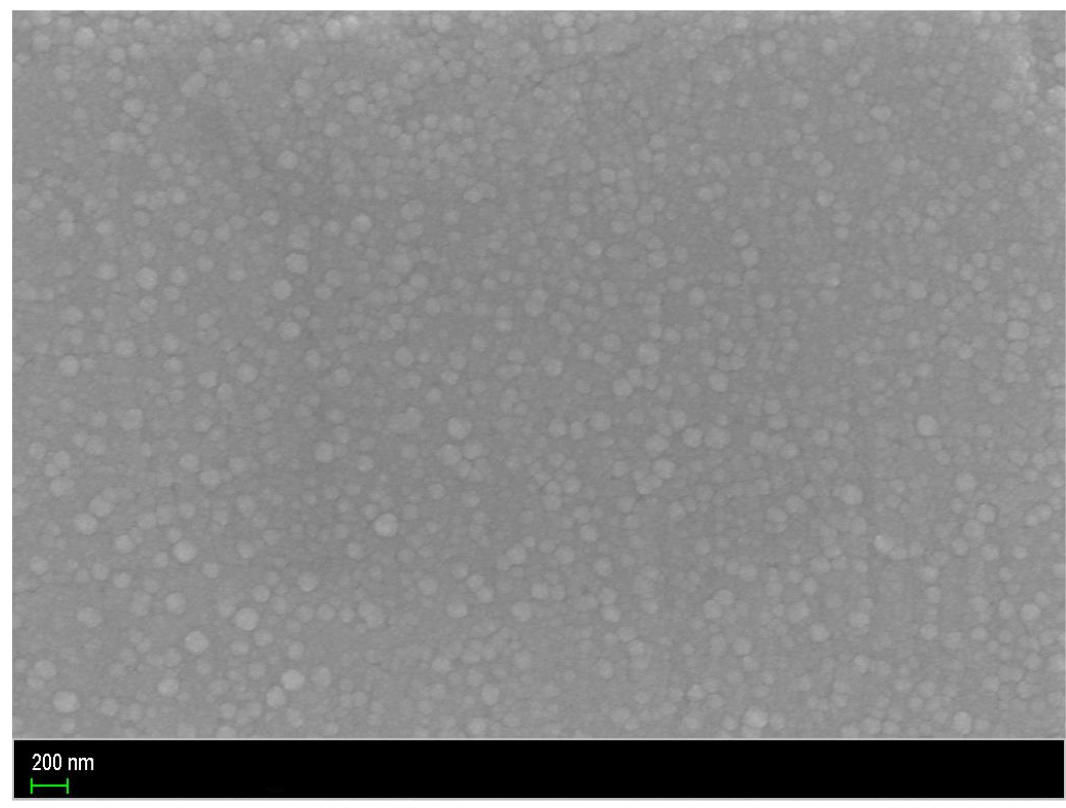

Figure S1. SEM image of the pure Si directly sputter-deposited on the Cu foil without TBNWs. As depositing of pure Si onto the surface of $\mathrm{Cu}$ foil without decorated of TBNWs as the scaffolds, only Si aggregates forming denser films could be observed, which may confront severe problems of crack and pulverization, finally leading to rapid capacity decay for the performances of the cell.

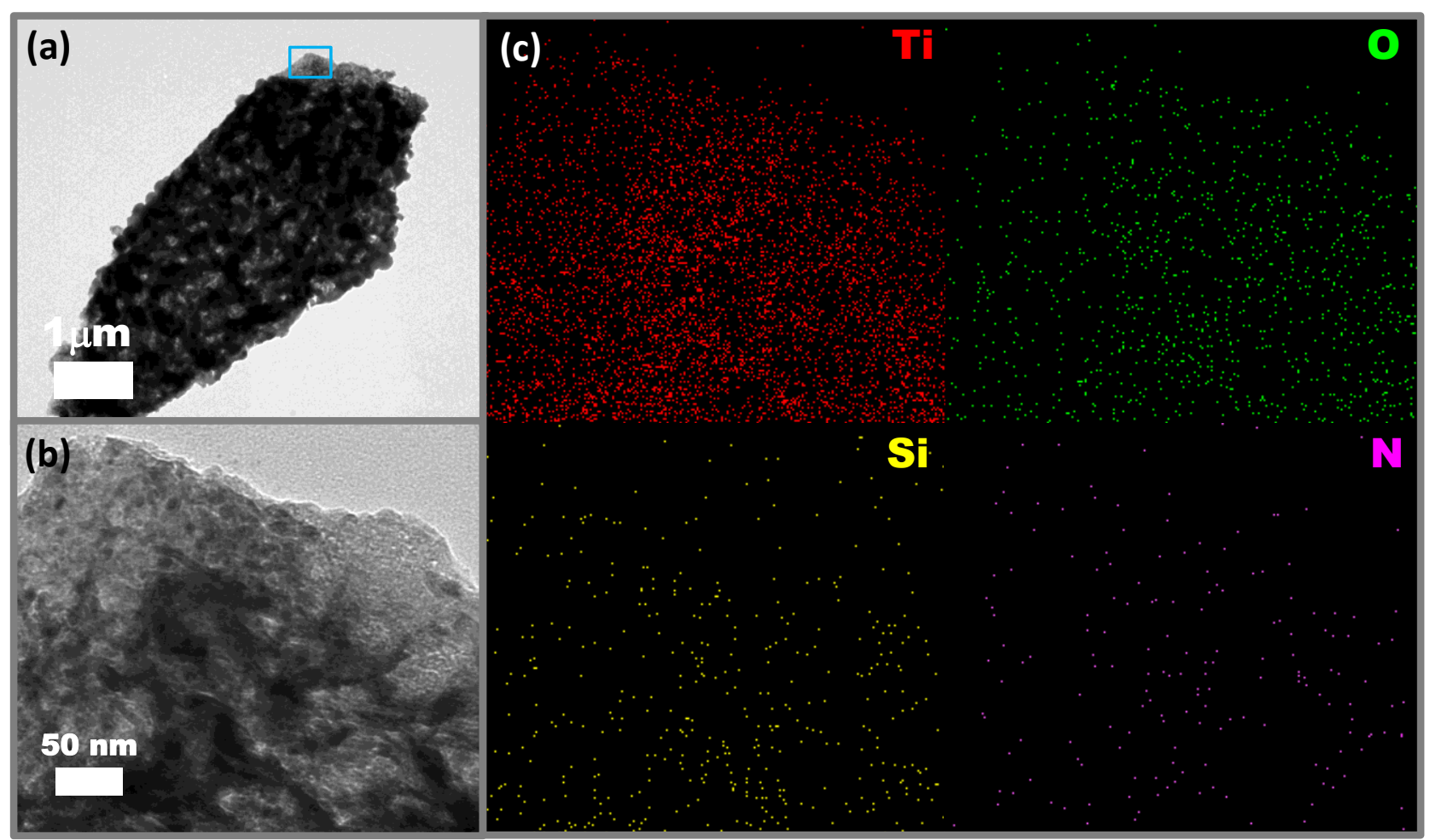

Figure S2. (a) Transmission electron microscopy (TEM) image of TCPP/Si/TBNW composite. (b) The TEM image take from the square-marked area and (c) its corresponding elemental mappings of titanium, oxygen, silicon, and nitrogen. The element mapping results indicated the successful formation of $\mathrm{TiO}_{2}, \mathrm{Si}$, and TCPP materials. 


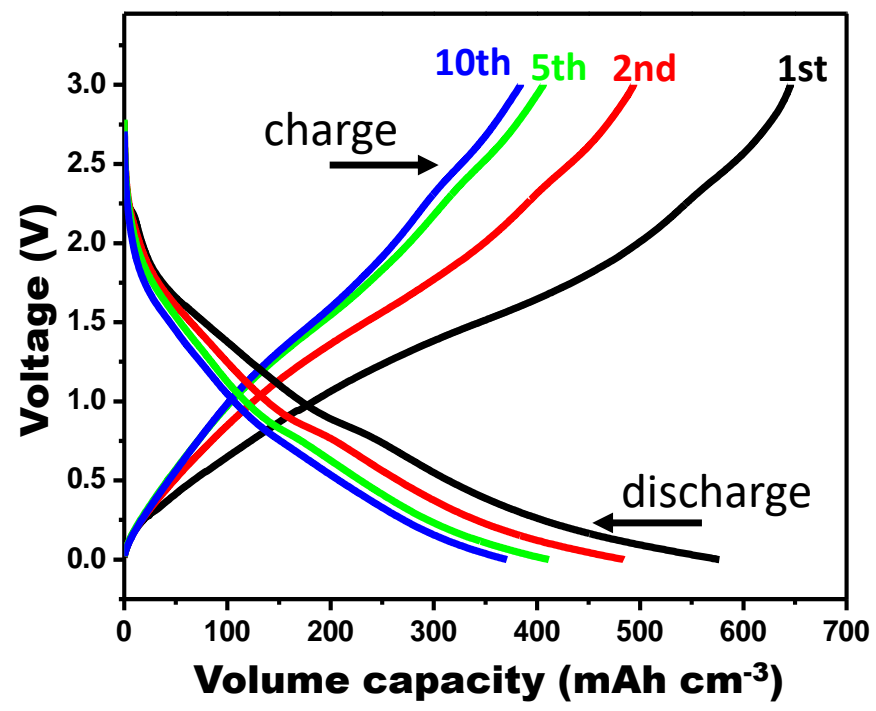

Figure S3. Typical charge-discharge profiles of the anatase-type TBNW anode at a rate of $0.1 \mathrm{C}(1 \mathrm{C}=$ $0.15 \mathrm{~mA} \mathrm{~cm}^{-1}$ ) between $0 \mathrm{~V}$ and $3 \mathrm{~V}$. For anatase-type $\mathrm{TiO}_{2}$ sample, a potential plateau located at approximately $1.8 \mathrm{~V}$ which is corresponding to the $\mathrm{Li}^{+}$ion intercalaction and deintercalation into the $\mathrm{TiO}_{2}$ lattice should be observed during the charge/discharge process. However, no characteristic features of the $\mathrm{TiO}_{2}$ in high potential region (>1.0 V) was found here, which could be ascribed to the shortened diffusion path of $\mathrm{Li}^{+}$ion during the intercalation/de-intercalation process for $1 \mathrm{D}$ nanowire architecture. ${ }^{2-3}$
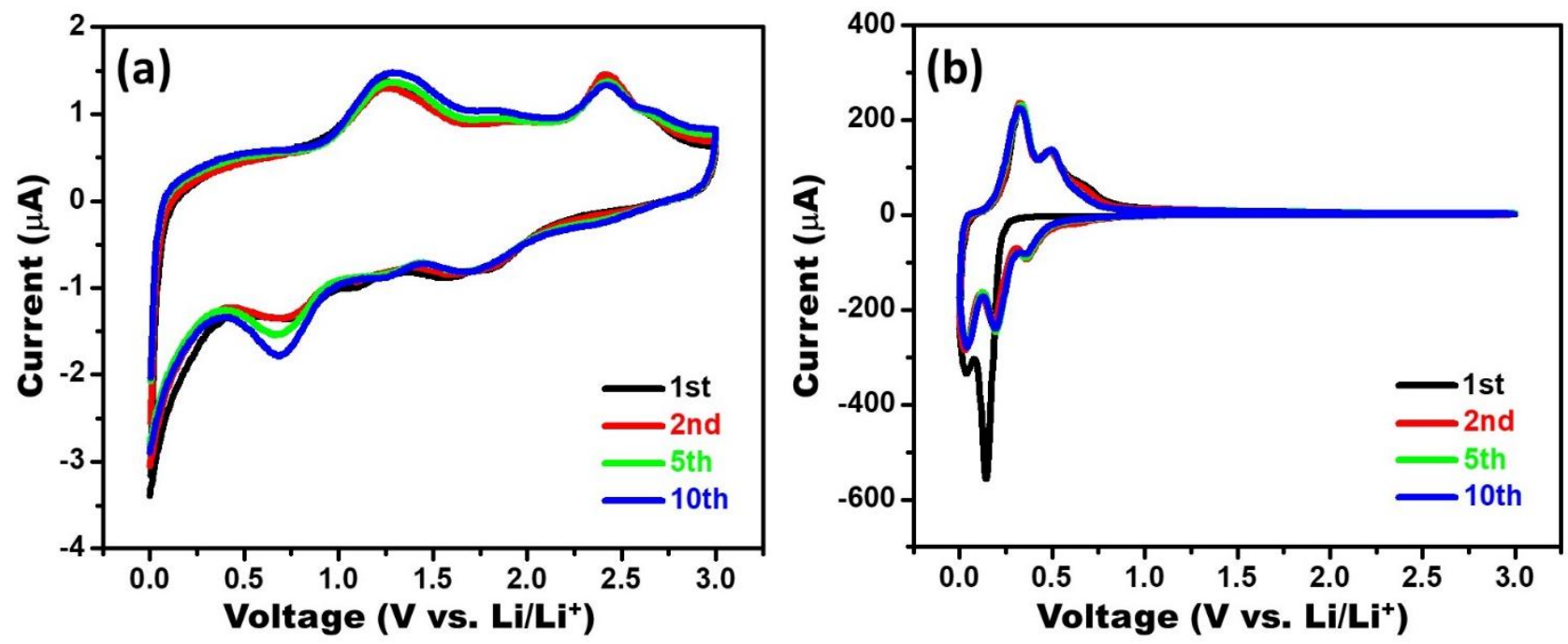

Figure S4. Typical cyclic voltammograms of (a) pure TCPP and (b) pure Si directly deposited onto the surface of $\mathrm{Cu}$ foils within the voltage range of $0-3 \mathrm{~V}$ versus $\mathrm{Li} / \mathrm{Li}^{+}$at a scan rate of $1 \mathrm{mV} \mathrm{s}{ }^{-1}$. In pure TCPP anode, the peaks in the first cathodic process appeared in high potential region $(>1.0 \mathrm{~V})$ can be attributed to the formation of solid electrolyte interface (SEI) film; the peak located around $0 \mathrm{~V}$ (from 0 to $0.4 \mathrm{~V}$ ) can be ascribed to the insertion of Li-ions into the $\pi$-conjugated system of TCPP. ${ }^{4}$ In pure $\mathrm{Si}$ anode, the peaks related to the $\mathrm{Si}-\mathrm{Li}$ alloying/dealloying reactions can be observed. Two cathodic peaks (lithiation) at 0.04 and $0.20 \mathrm{~V}$ and two anodic peaks (delithiation) at 0.32 and $0.50 \mathrm{~V}$ can be obtained, which correspond to the conversion of amorphous Si to $\mathrm{Li}_{x} \mathrm{Si}$ alloy and the delithiation of $\mathrm{Li}_{x} \mathrm{Si}$ back to amorphous $\mathrm{Si}$, respectively, consistent with the previous report. ${ }^{5}$ 


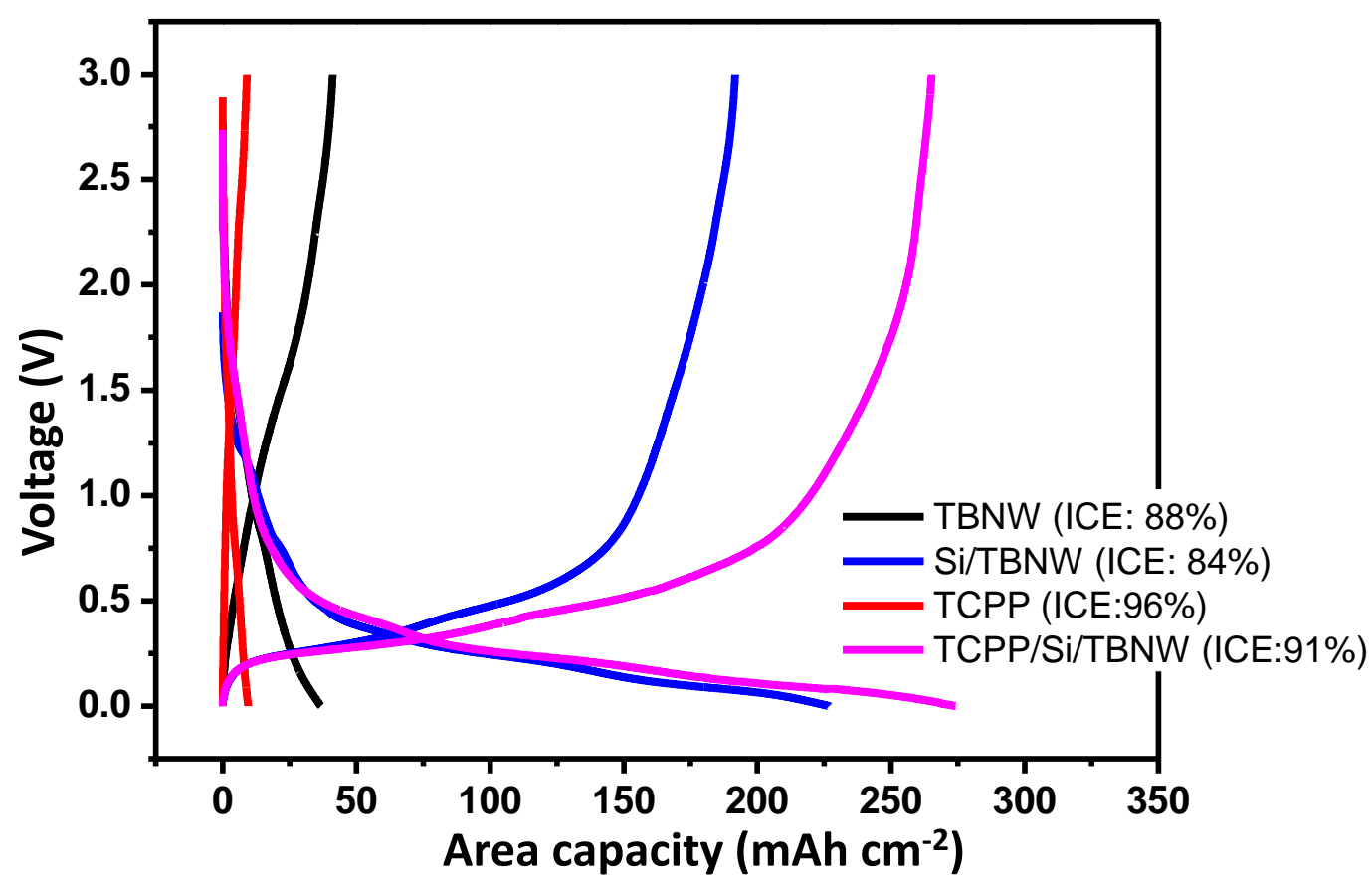

Figure S5. Typical initial charge-discharge profiles of the TBNW, Si/TBNW, pure TCPP, and TCPP/Si/TBNW anodes at a rate of $0.1 \mathrm{C}\left(1 \mathrm{C}=0.15 \mathrm{~mA} \mathrm{~cm}^{-1}\right)$ between $0 \mathrm{~V}$ and $3 \mathrm{~V}$. The Si/TBNW showed lower coulombic efficiency of $\sim 84 \%$. At the same time, the coulombic efficiency of the pure TCPP was about $96 \%$. Thus, combining of Si/TBNW and TCPP will be an effective way to realize the high coulombic efficiency. As can be seen, compared with the Si/TBNW, the TCPP/Si/TBNW composite anode presented an increased coulombic efficiency of $\sim 91 \%$.

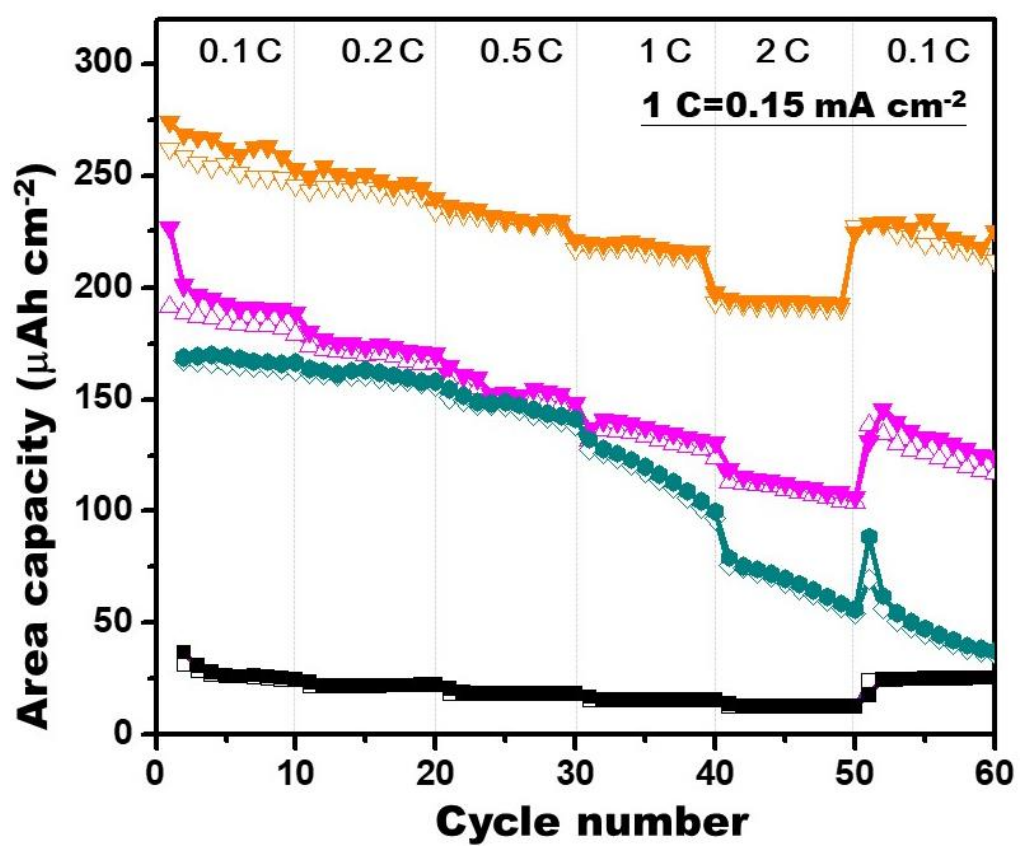

Figure S6. Charge-discharge area capacities at different rates of (orange line) TCPP/Si/TBNW composite, (purple line) Si/TBNW, (green line) pure Si, and (black line) TBNW anodes with the voltage window between $0 \mathrm{~V}$ to $3.0 \mathrm{~V} \mathrm{vs}$. $\mathrm{Li} / \mathrm{Li}^{+}$. 

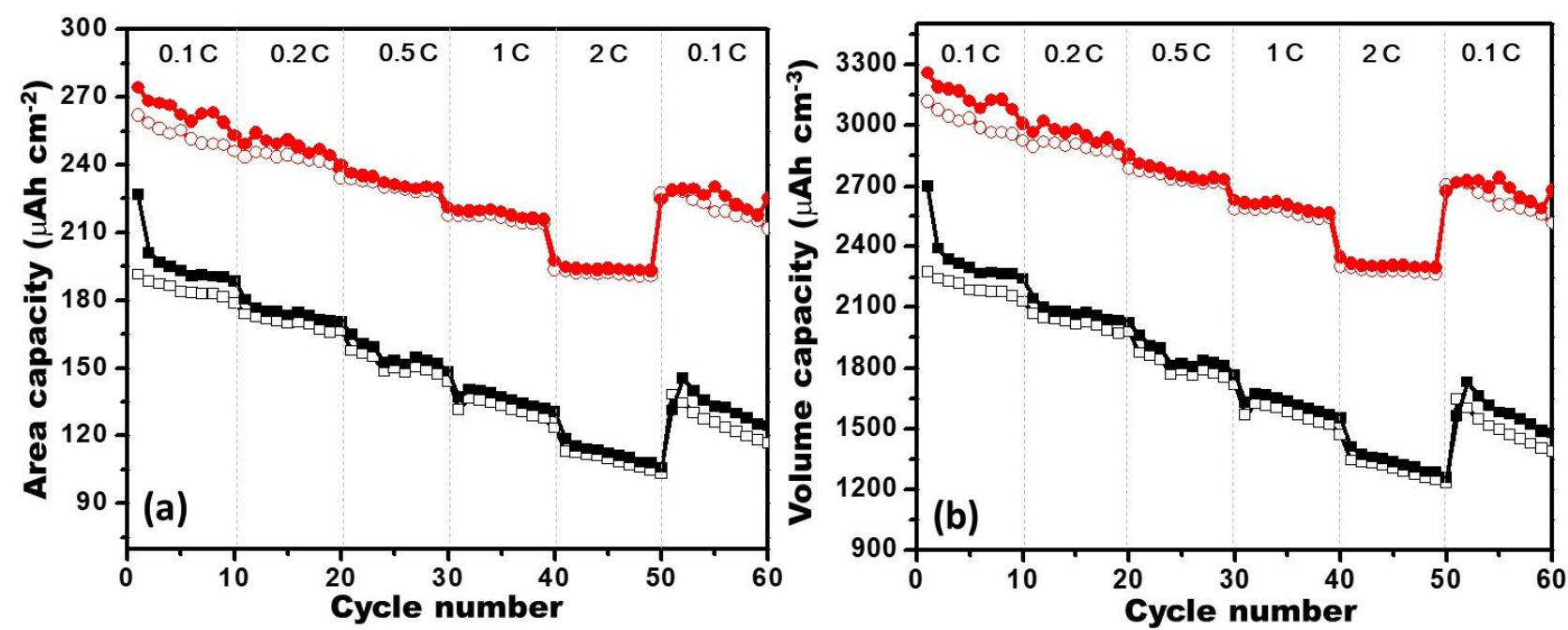

Figure S7 Charge-discharge area capacities (a) and volume capacities (b) at different rates of $\mathrm{TCPP} / \mathrm{Si} / \mathrm{TBNW}$ composite anode in the voltage window of $0 \mathrm{~V}-3.0 \mathrm{~V} \mathrm{vs}$. $\mathrm{Li}^{\mathrm{L}} \mathrm{Li}^{+}$.

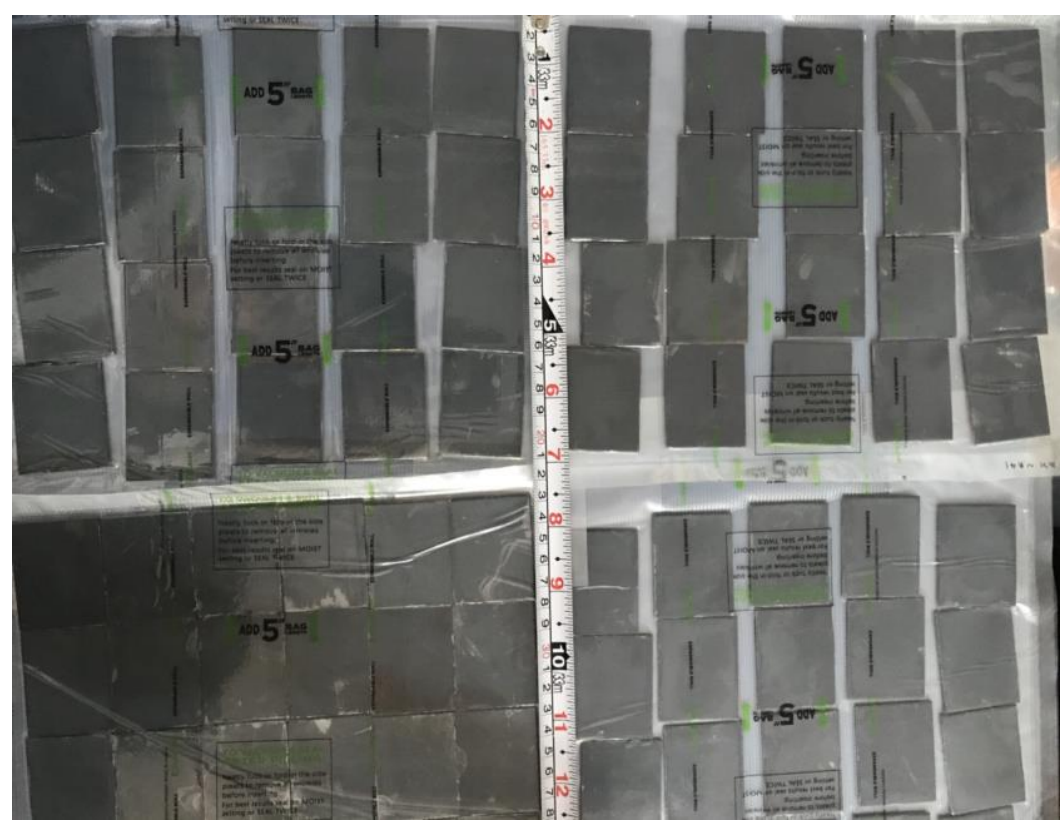

Figure S8. Industrial scale production of the silicon-porphyrin web-nanowire based anodes with the size of $4 \mathrm{~cm} \times 5 \mathrm{~cm}$ that we can make 120 pieces a day.

\section{References:}

(1) Chen, J.-Z.; Ko, W.-Y.; Yen, Y.-C.; Chen, P.-H.; Lin, K.-J., Hydrothermally Processed TiO2 Nanowire Electrodes with Antireflective and Electrochromic Properties. ACS Nano 2012, 6, 6633-6639.

(2) Wagemaker, M.; Borghols, W. J. H.; Mulder, F. M., Large Impact of Particle Size on Insertion Reactions. A Case for Anatase LixTiO2. 2007, 129, 4323-4327.

(3) Luo, Y.; Zhang, Y.; Huang, J., A hierarchically structured anatase-titania/indium-tin-oxide nanocomposite as an anodic material for lithium-ion batteries. 2017, 19, 6972-6978.

(4) Wu, H.; Zhang, J.; Du, X.; Zhang, M.; Yang, J.; Zhang, J.; Luo, T.; Liu, H.; Xu, H.; Cui, G., A large $\pi-$ conjugated tetrakis (4-carboxyphenyl) porphyrin anode enables high specific capacity and superior 
cycling stability in lithium-ion batteries. 2019, 55, 11370-11373.

(5) Ma, T.; Yu, X.; Li, H.; Zhang, W.; Cheng, X.; Zhu, W.; Qiu, X., High Volumetric Capacity of Hollow Structured SnO2@Si Nanospheres for Lithium-Ion Batteries. Nano Lett. 2017, 17, 3959-3964. 